\title{
\begin{tabular}{l|l} 
Mibraries & DSpace@MIT
\end{tabular}
}

\author{
MIT Open Access Articles
}

\section{A model for the critical voltage for electrical degradation of GaN high electron mobility transistors}

The MIT Faculty has made this article openly available. Please share how this access benefits you. Your story matters.

Citation: Jungwoo Joh, and J.A. del Alamo. “Critical Voltage for Electrical Degradation of GaN High-Electron Mobility Transistors." Electron Device Letters, IEEE 29.4 (2008): 287-289. (C) 2008 IEEE

As Published: http://dx.doi.org/10.1109/LED.2008.917815

Publisher: Institute of Electrical and Electronics Engineers

Persistent URL: http://hdl.handle.net/1721.1/59403

Version: Final published version: final published article, as it appeared in a journal, conference proceedings, or other formally published context

Terms of Use: Article is made available in accordance with the publisher's policy and may be subject to US copyright law. Please refer to the publisher's site for terms of use. 


\title{
Critical Voltage for Electrical Degradation of GaN High-Electron Mobility Transistors
}

\author{
Jungwoo Joh and Jesús A. del Alamo
}

\begin{abstract}
We have found that there is a critical drain-to-gate voltage beyond which GaN high-electron mobility transistors start to degrade in electrical-stress experiments. The critical voltage depends on the detailed voltage biasing of the device during electrical stress. It is higher in the OFF state and high-power state than at $V_{\mathrm{DS}}=0$. In addition, as $\left|V_{\mathrm{GS}}\right|$ increases, the critical voltage decreases. We have also found that the stress current does not affect the critical voltage although soft degradation at low voltages takes place at high stress currents. All of our findings are consistent with a degradation mechanism based on crystallographic-defect formation due to the inverse piezoelectric effect. Hot-electron-based mechanisms seem to be in contradiction with our experimental results.
\end{abstract}

Index Terms-DC stress, degradation, GaN, high-electron mobility transistor (HEMT), reliability.

\section{INTRODUCTION}

$\mathbf{O}$ NE OF THE MOST promising devices for RF power amplifier and high-voltage switching applications is the GaN high-electron mobility transistor (HEMT). Due to the extraordinary material properties of GaN, GaN HEMTs have already shown an output power density over $30 \mathrm{~W} / \mathrm{mm}$ at $8 \mathrm{GHz}$ [1]. Recently, GaN-based HEMTs have also expanded their frequency range. A power density of $2.1 \mathrm{~W} / \mathrm{mm}$ has been demonstrated in W-band [2]. However, in spite of this impressive performance, a wide-scale deployment of GaN HEMTs has yet to take place. The main impediment is the limited electrical reliability that these devices exhibit.

Although a few studies have been conducted attempting to provide understanding of the physics behind the electrical degradation of GaN HEMTs [3]-[7], detailed failure mechanisms have yet to be identified. The prevailing hypothesis in the literature is hot-electron-induced trap generation [3], [4]. Recently, we have presented experimental evidence that is in contradiction with this hypothesis [7]. We have also proposed a degradation mechanism based on crystallographic-defect formation through the inverse piezoelectric effect [7]. In this letter, we study in detail one of our most significant findings in [7]: the observation of a critical voltage for electrical degradation. We show that the critical voltage depends on the bias configuration

Manuscript received November 12, 2007; revised January 16, 2008. This work was supported by the Army Research Laboratory (ARL) under Contract W911QX-05-C-0087. The review of this letter was arranged by Editor G. Meneghesso.

The authors are with the Microsystems Technology Laboratories, Massachusetts Institute of Technology, Cambridge, MA 02139 USA (e-mail: jungwoo@mit.edu).

Color versions of one or more of the figures in this letter are available online at http://ieeexplore.ieee.org.

Digital Object Identifier 10.1109/LED.2008.917815

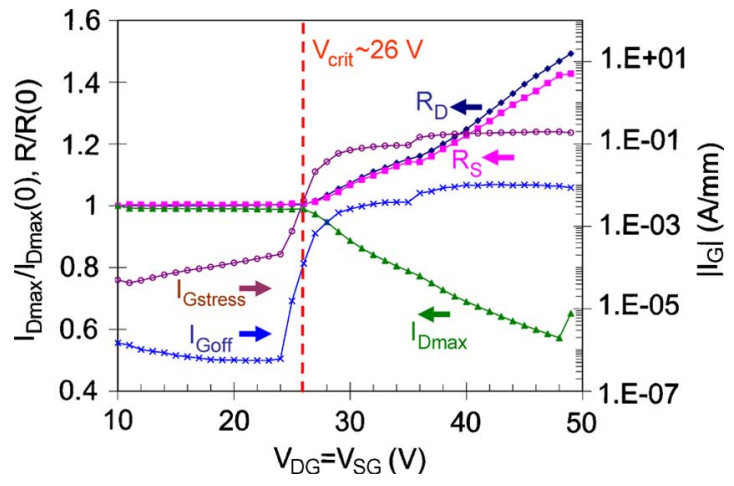

Fig. 1. Change in normalized $I_{\mathrm{D} \max }, R_{D}, R_{S}, I_{\mathrm{Gstress}}$, and $I_{\mathrm{Goff}}$ as a function of stress voltage in a step-stress experiment in the $V_{\mathrm{DS}}=0$ state $\left(V_{\mathrm{DG}}=10-50 \mathrm{~V}\right.$ in $1-\mathrm{V}$ steps $)$.

during stress and on the device geometry in ways that are inconsistent with a hot-electron hypothesis.

\section{EXPERIMENTAL RESULT}

The devices that we have studied are millimeter-wave industrial GaN HEMTs of $L_{G}=0.25 \mu \mathrm{m}$ with a field plate. Both source-to-gate gap and gate-to-drain gap distances were $2 \mu \mathrm{m}$. A typical device shows $I_{\mathrm{D} \max }$ of $1.2 \mathrm{~A} / \mathrm{mm}, V_{T}$ of $-3 \mathrm{~V}$, and $f_{T}$ of $40 \mathrm{GHz}$. We have electrically stressed these devices in dc at room temperature and in $\mathrm{N}_{2}$ under various conditions. During stress, we have measured several device parameters by a benign characterization suite [8].

First, we carried out step-stress experiments in the $V_{\mathrm{DS}}=0$ state. This condition is interesting in that no current flows through the channel of the device, and both sides of the device can be stressed simultaneously. In a typical experiment, $V_{\mathrm{GS}}$ is stepped from -10 to $-50 \mathrm{~V}$ in $1-\mathrm{V}$ steps while keeping $V_{\mathrm{DS}}=0$. The device is stressed for $1 \mathrm{~min}$ in each voltage step. Fig. 1 shows the change in $I_{\mathrm{D} \max }$ (drain current at $V_{\mathrm{DS}}=5 \mathrm{~V}$ and $V_{\mathrm{GS}}=2 \mathrm{~V}$ ), $R_{S}, R_{D}$, stress gate current, and $I_{\mathrm{Goff}}$ (gate current at $V_{\mathrm{DS}}=0.1 \mathrm{~V}$ and $V_{\mathrm{GS}}=-5 \mathrm{~V}$ ) as the stress experiment proceeds. There is a negligible degradation up to around $V_{\mathrm{DG}}=V_{\mathrm{SG}}=26 \mathrm{~V}$. At this critical voltage, degradation in all figures of merit starts sharply and increases as the stress experiment proceeds.

Our finding of a critical voltage for electrical degradation is surprising in itself. More surprising is realizing that this critical voltage is quite low since, separately, we have verified that these devices operate normally at $30 \mathrm{~V}$ with minor degradation [9]. In order to study this, we have carried out similar experiments under other bias conditions: high-power state and OFF state. 

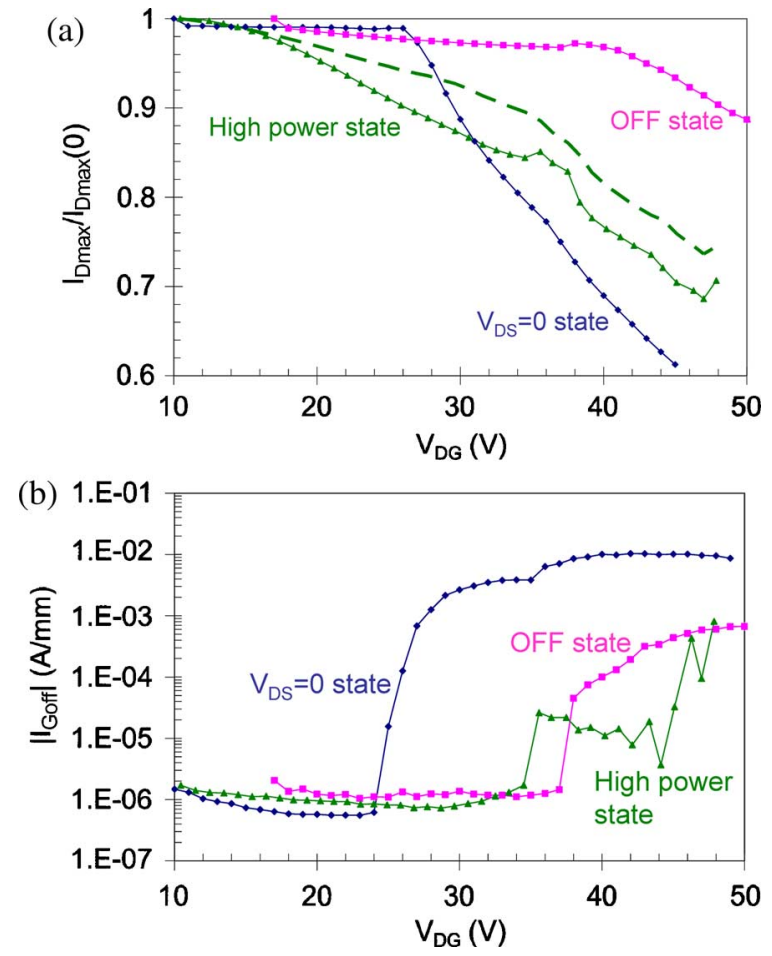

Fig. 2. (a) Change in normalized $I_{\mathrm{D} \max }$ in step-stress experiments for three different stress conditions. Dashed line represents the estimated change in $I_{\mathrm{D} \text { max }}$ in the high-power state removing the effect of $V_{T}$ change. (b) Change in the gate leakage current $I_{\text {Goff }}$ (gate current at $V_{\mathrm{DS}}=0.1 \mathrm{~V}$ and $V_{\mathrm{GS}}=$ $-5 \mathrm{~V}$ ) in the same experiment.

Fig. 2(a) shows the $I_{\mathrm{D} \max }$ degradation in three different stepstress experiments. In the OFF state, the stress bias conditions were $V_{\mathrm{GS}}=-7 \mathrm{~V}$ and $V_{\mathrm{DS}}=10-50 \mathrm{~V}$. In the high-power state, we applied $V_{\mathrm{DS}}=10-50 \mathrm{~V}$ with $I_{D}=0.8 \mathrm{~A} / \mathrm{mm}$. For reference, we replot the data for $V_{\mathrm{DS}}=0$ from Fig. 1. In all these experiments, the devices have been stressed for $1 \mathrm{~min}$ in each step, and the voltage step size was $1 \mathrm{~V}$. In all three experiments, we observe again a mirror image behavior in the degradation of $I_{\mathrm{D} \text { max }}$ and $R_{D}$ (not shown).

In the OFF state, we observe a well-defined critical voltage of about $38 \mathrm{~V}$. In the high-power state, we observe a gentle degradation at low $V_{\mathrm{DS}}$ followed by an increase in the slope of degradation at around $35 \mathrm{~V}$. The low-level degradation in $I_{\mathrm{D} \max }$ at low $V_{\mathrm{DG}}$ in the high-power state is partly due to a significant positive shift of threshold voltage (about $+0.5 \mathrm{~V}$ for the entire experiment). The $I_{\mathrm{D} \max }$ change that would have taken place if there had been no $V_{T}$ change is shown through the dashed line in Fig. 2(a). As it can be seen, for $V_{\mathrm{DG}}>30 \mathrm{~V}$, the overall degradation in the high-power state is lower than in the $V_{\mathrm{DS}}=0$ state in spite of the high current. This is inconsistent with a hotelectron hypothesis [3], [4].

Fig. 2(b) shows the time evolution of $I_{\mathrm{Goff}}$ for these experiments. In all cases, $I_{\text {Goff }}$ exhibits a sharp rise at around the same critical voltage of Figs. 1 and 2(a), which implies that the $I_{D}$ and $I_{G}$ degradations have the same origin [10]. This behavior in $I_{G}$ again confirms that the critical voltages in the high-power state and in the OFF state are about 10 and $15 \mathrm{~V}$ higher, respectively, than that in the $V_{\mathrm{DS}}=0$ state.

In principle, this difference in critical voltage could result from different values of $V_{\mathrm{GS}}$ or different levels of stress current.
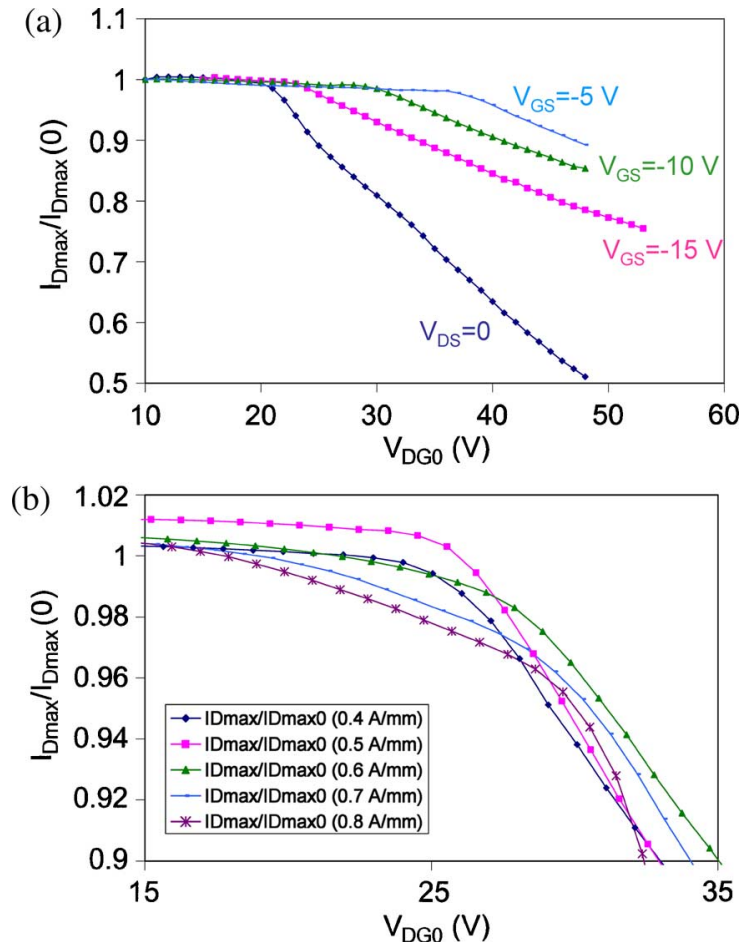

Fig. 3. Change in $I_{\mathrm{D} \max }$ in two different sets of step-stress experiments. (a) Impact of $V_{\mathrm{GS}}$ on the critical voltage $V_{\mathrm{DG}}$ crit. $V_{\mathrm{GS}}=-5,-10$, and $-15 \mathrm{~V}$ were investigated. For the $V_{\mathrm{DS}}=0$ state, $V_{\mathrm{GS}}=-V_{\mathrm{DG}}$. $V_{\mathrm{DG}}$ crit increases as $\left|V_{\mathrm{GS}}\right|$ decreases. (b) Impact of stress current $(0.4-0.8 \mathrm{~A} / \mathrm{mm})$. No clear impact of the stress current is observed.

We have studied both possibilities. We investigated the impact of $V_{\mathrm{GS}}$ by step stressing $V_{\mathrm{DS}}$ with $V_{\mathrm{GS}}$ fixed at different values. The results are shown in Fig. 3(a) with the data for the $V_{\mathrm{DS}}=0$ state as reference (in this case, $V_{\mathrm{GS}}=-V_{\mathrm{DG}}$ ). As one can see, $V_{\mathrm{DG} \text { crit }}$ increases as $\left|V_{\mathrm{GS}}\right|$ decreases. This change in $V_{\mathrm{DG}}$,rit does not come from a change in current. For $V_{\mathrm{GS}}=-10$ and $-15 \mathrm{~V}$, the stress current was less than $1 \mathrm{~mA} / \mathrm{mm}$ up to $V_{\text {DGcrit }}$. Even for $V_{\mathrm{GS}}=-5 \mathrm{~V}$, the stress current was $115 \mathrm{~mA} / \mathrm{mm}$ at $V_{\text {DGcrit }}$. Further confirmation of the relatively unimportant role of current comes from the step-stress experiments with current as a parameter. In Fig. 3(b), we can see that the stress current has a little impact on $V_{\text {DGcrit }}$ except for a softening of the corner at high current, just as in the high-power state.

The results that we show in this letter have been observed with great reproducibility in many different devices fabricated through different processes on different heterostructures.

\section{DISCUSSION}

In [7], we proposed a degradation mechanism for $\mathrm{GaN}$ HEMT that is based on crystallographic-defect formation through the inverse piezoelectric effect due to high vertical electric field at the drain edge of the gate. In this hypothesis, the mechanical strain produced by this electric field adds on top of the tensile strain due to lattice mismatch between AlGaN barrier and $\mathrm{GaN}$ layer and increases the elastic energy in the AlGaN barrier. If this elastic energy exceeds a critical value, crystallographic defects are formed which become trapping sites for electrons. The elastic energy in the AlGaN barrier layer is proportional to strain squared, and strain is linearly 
proportional to the vertical electric field which is a function of voltage. The existence of a critical voltage for electrical degradation is consistent with the notion that lattice damage does not occur until the elastic energy reaches its critical value [11].

A higher $V_{\text {DGcrit }}$ in the OFF state and in the high-power state when compared with the $V_{\mathrm{DS}}=0$ state as well as the $V_{\mathrm{GS}}$ dependence of $V_{\mathrm{DGcrit}}$ can be explained by the impact of strain field produced from the source side of the device. As $V_{\mathrm{SG}}$ increases, the strain field at the source side of the gate edge increases, and the total elastic-energy density under the gate increases, resulting in lower $V_{\text {DGcrit }}$.

This hypothesis also explains the gate-length dependence of $V_{\text {DGcrit }}$ in the $V_{\mathrm{DS}}=0$ state that we reported in [7]. There, we found that $V_{\mathrm{DGcrit}}$ decreases as $L_{G}$ decreases with stress in the $V_{\mathrm{DS}}=0$ state. This result is also inconsistent with a hotelectron-related hypothesis [3], [4] in that to the first order, the electrostatics in the extrinsic portion of the device must be the same regardless of the value of $L_{G}$ if the gate-drain gap length does not change, as is the case in these devices. Therefore, at a given bias point, the hot-electron production should be about the same in all these devices. In contrast with this, we have not seen any clear dependence of degradation on $L_{G}$ in the OFF state and the high-power state. In our hypothesis, in the $V_{\mathrm{DS}}=0$ state, longer gate-length devices bring the two highfield points at both ends of the gate further apart, presumably resulting in an overall lower strain in the AlGaN layer under the gate. In the OFF state or in the high-power state, the gate length should have less impact on the critical voltage as the mechanical stress from the source side is smaller. Along with these results, the small impact of current on $V_{\mathrm{DG}}$ rit that we observe confirms that the role of hot electrons is minor since hot-electron effects should depend on current and $V_{\mathrm{DG}}$.

On top of the strain-related mechanism, some other degradation mechanism seems to be involved for high currents for which we observe a soft degradation below the critical voltage [Fig. 3(b)]. This may be due to an increased channel temperature or some hot-electron effect. In fact, we also observe that in the high-power state, the source resistance degrades to some extent. This needs to be investigated further in the future.

\section{CONCLUSION}

We have found a critical voltage for electrical degradation beyond which a GaN HEMT starts to degrade abruptly. We have found that $V_{\mathrm{DGcrit}}$ is higher in the OFF state and the high-power state than in the $V_{\mathrm{DS}}=0$ state. This is due to the fact that high $V_{\mathrm{SG}}$ decreases $V_{\mathrm{DGcrit}}$. We have also found that the stress current does not change $V_{\mathrm{DG}}$. These results are consistent with earlier observations of higher $V_{\mathrm{DG}}$ crit in longer devices in the $V_{\mathrm{DS}}=0$ condition, but being gate length independent in the other bias conditions. Our experimental results are consistent with a defect formation mechanism due to the inverse piezoelectric effect [7].

\section{ACKNOWLEDGMENT}

This research took place at the Microsystems Technology Laboratories, MIT. The authors would like to thank TriQuint Semiconductor and BAE Systems for their collaborative efforts.

\section{REFERENCES}

[1] Y. F. Wu, A. Saxler, M. Moore, R. P. Smith, S. Sheppard, P. M. Chavarkar, T. Wisleder, U. K. Mishra, and P. Parikh, " 30 W/mm GaN HEMTs by field plate optimization," IEEE Electron Device Lett., vol. 25, no. 3, pp. 117119, Mar. 2004.

[2] M. Micovic, A. Kurdoghilian, P. Hashimoto, M. Hu, M. Antcliffe, P. J. Willadsen, W. S. Wong, R. Bowen, I. Milosavljevic, A. Schmitz, Y. Yoon, M. Wetzel, and D. H. Chow, "GaN HFET for W-band power applications," in IEDM Tech. Dig., 2006, pp. 425-427.

[3] H. Kim, V. Tilak, B. M. Green, J. A. Smart, W. J. Schaff, J. R. Shealy, and L. F. Eastman, "Reliability evaluation of high power AlGaN/GaN HEMTs on SiC substrate," Phys. Stat. Sol. A, vol. 188, no. 1, pp. 203-206, Nov. 2001.

[4] A. Sozza, C. Dua, E. Morvan, M. A. diForte-Poisson, S. Delage, F. Rampazzo, A. Tazzoli, F. Danesin, G. Meneghesso, E. Zanoni, A. Curutchet, N. Malbert, N. Labat, B. Grimbert, and J.-C. De Jaeger, "Evidence of traps creation in GaN/AlGaN/GaN HEMTs after a 3000 hour on-state and off-state hot-electron stress," in IEDM Tech. Dig., 2005, pp. 590-593.

[5] D. Pavlidis, P. Valizadeh, and S. H. Hsu, "AlGaN/GaN high electron mobility transistor (HEMT) reliability," in Proc. GaAs Symp., 2005, pp. 265-268.

[6] C. Lee, L. Witkowski, H.-Q. Tserng, P. Saunier, R. Birkhahn, D. Olson, D. Olson, G. Munns, S. Guo, and B. Albert, "Effects of AlGaN/GaN HEMT structure on RF reliability," Electron. Lett., vol. 41, no. 3, pp. 155157, Feb. 2005.

[7] J. Joh and J. A. del Alamo, "Mechanisms for electrical degradation of GaN high-electron mobility transistors," in IEDM Tech. Dig., 2006, pp. 415-418.

[8] J. Joh, "Degradation mechanisms of GaN high electron mobility transistors," M.S. thesis, MIT, Cambridge, MA, 2007.

[9] J. L. Jimenez, U. Chowdhury, M. Y. Kao, T. Balistreri, C. Lee, P. Saunier, P. C. Chao, W. W. Hu, K. Chu, A. Immorlica, J. A. del Alamo, J. Joh, and M. Shur, "Failure analysis of X-band GaN FETs," presented at the Reliability Compound Semiconductors Workshop (ROCS), San Antonio, TX, 2006.

[10] J. Joh, L. Xia, and J. A. del Alamo, "Gate current degradation mechanisms of GaN high electron mobility transistors," in IEDM Tech. Dig., 2007, pp. 385-388.

[11] S. R. Lee, D. D. Koleske, K. C. Cross, J. A. Floro, K. E. Waldrip, A. T. Wise, and S. Mahajan, "In situ measurements of the critical thickness for strain relaxation in AlGaN/GaN heterostructures," Appl. Phys. Lett., vol. 85 , no. 25 , pp. 6164-6166, Dec. 2004. 